ARTICLE

Received 13 Oct 2013 | Accepted 2 Jun 2014 | Published 10 Jul 2014

DOI: $10.1038 /$ ncomms5276

\title{
CHOLINE TRANSPORTER-LIKE1 is required for sieve plate development to mediate long-distance cell-to-cell communication
}

Jan Dettmer ${ }^{1, \star}$, Robertas Ursache $^{2, \star}$, Ana Campilho ${ }^{3, \star}$, Shunsuke Miyashima², llya Belevich ${ }^{2}$, Seana O'Regan ${ }^{4}$, Daniel Leroy Mullendore ${ }^{5}$, Shri Ram Yadav ${ }^{2}$, Christa Lanz ${ }^{6}$, Luca Beverina ${ }^{7}$, Antonio Papagni ${ }^{7}$, Korbinian Schneeberger ${ }^{8}$, Detlef Weigel ${ }^{6}$, York-Dieter Stierhof ${ }^{9}$, Thomas Moritz ${ }^{10}$, Michael Knoblauch ${ }^{5}$, Eija Jokitalo ${ }^{2} \&$ Ykä Helariutta ${ }^{2}$

Phloem, a plant tissue responsible for long-distance molecular transport, harbours specific junctions, sieve areas, between the conducting cells. To date, little is known about the molecular framework related to the biogenesis of these sieve areas. Here we identify mutations at the CHERT/AtCTL1 locus of Arabidopsis thaliana. The mutations cause several phenotypic abnormalities, including reduced pore density and altered pore structure in the sieve areas associated with impaired phloem function. CHER 1 encodes a member of a poorly characterized choline transporter-like protein family in plants and animals. We show that CHER1 facilitates choline transport, localizes to the trans-Golgi network, and during cytokinesis is associated with the phragmoplast. Consistent with its function in the elaboration of the sieve areas, CHER1 has a sustained, polar localization in the forming sieve plates. Our results indicate that the regulation of choline levels is crucial for phloem development and conductivity in plants.

\footnotetext{
${ }^{1}$ Cell Biology Division, Department of Biology, University of Erlangen-Nuremberg, 91058 Erlangen, Germany. ${ }^{2}$ Institute of Biotechnology, Department of Biological and Environmental Sciences, University of Helsinki, Helsinki FIN-00014, Finland. ${ }^{3}$ Institute for Molecular and Cell Biology (IBMC), University of Porto, Porto 4150-180, Portugal. ${ }^{4}$ Neurophotonics Laboratory, CNRS/Université Paris Descartes, 45, rue des Saints-Pères, 75270 Paris, France. ${ }^{5}$ School of Biological Sciences, Washington State University, Pullman, Washington 99164-4236, USA. ${ }^{6}$ Department of Molecular Biology, Max Planck Institute for Developmental Biology, 72076 Tuebingen, Germany. ${ }^{7}$ Department of Materials Science, University of Milano-Bicocca, Via R. Cozzi 55, 20125 Milano, Italy. ${ }^{8}$ Max Planck Institute for Plant Breeding Research, Department for Plant Developmental Biology, 50829 Cologne, Germany. ${ }^{9}$ ZMBP, Mikroskopie, Universität Tübingen, Auf der Morgenstelle 5, 72076 Tübingen, Germany. ${ }^{10}$ Department of Forest Genetics and Plant Physiology, Umeå Plant Science Center, Swedish University of Agricultural Sciences, SE-90183 Umeå, Sweden. ${ }^{\star}$ These authors contributed equally to this work. Correspondence and requests for materials should be addressed to Y.H. (email: yrjo.helariutta@helsinki.fi).
} 
$\mathrm{M}$ ulticellular organisms have diverse cellular structures to facilitate cell communication, such as receptors, channels and junction structures. Choline metabolism and transport has been known for a long time as a major factor for cell communication in animals. Choline is classified as an essential nutrient for humans and serves as a precursor for the synthesis of both signalling and major membrane phospholipids, as well as (glycine) betaine and the neurotransmitter acetylcholine. Interference with choline uptake, transport or metabolism has been implicated in severe developmental defects and neurological disorders like Alzheimer and Parkinson's ${ }^{1}$. Interand intracellular choline transport are mediated by different transporter systems such as the polyspecific organic cation transporters, the high-affinity choline transporters (CHTs) and the intermediate-affinity choline transporter-like proteins $(\mathrm{CTLs})^{2,3}$. CHT1 is almost exclusively expressed in cholinergic neurons and is required for the reuptake of choline from the synaptic cleft into presynaptic neurons ${ }^{4-7}$, whereas the ubiquitous expression of CTL1/SLC44A1 in mammalian tissues suggest that choline transport is also required for more general cellular functions like phospholipid biosynthesis ${ }^{3,8-12}$. Interestingly, it has recently been suggested that CTL4/SLC44A4 may have a specific role in the synthesis of non-neuronal acetylcholine ${ }^{13}$.

Little is known about choline transport in plants. Early studies using isotope-labelled choline indicated that the compound can be transported by carriers into plant cells. Also phosphocholine, a compound derived from choline, is found in xylem exudate. This organic phosphate ester might serve for phosphate and nitrogen storage and is distributed by the vascular system throughout the plant $^{94,15}$. The idea that choline can be taken up from the media and distributed within the plant is further supported by the observation that externally supplied choline can rescue the developmental defects of xipotl, a phosphocholine biosynthesis mutant ${ }^{16}$. Choline might also be transported by the proline transporter BvBet/ProT1, which is expressed in phloem and xylem parenchyma cells of sugar beet ${ }^{17}$. Furthermore, observations made in bean (Phaseolus vulgaris) and soybean (Glycine max) suggest that extracellular choline is important for plant-pathogen interactions ${ }^{18}$.

The conducting tissues for long-distance transport in plants are xylem and phloem (Fig. 1a). Phloem is required for the distribution of photosynthetic products, hormones, RNA, peptides and other molecules from source to sink tissues. The phloem tissue consists of phloem parenchyma cells, sieve tube elements and companion cells (CCs). Transport occurs through sieve tube elements, narrow elongated cylindrical cells, adjusted to each other and forming a network spanning the entire length of the plant. The cell walls between connecting sieve elements (SEs), called sieve plates, are perforated by a large number of pores, enlarged plasmodesmata, which enable intercellular transport and communication (Fig. 1b). Three major components represent the simplified structure of plasmodesmata: a tube that establishes plasma membrane continuity between adjacent cells, a central axial element derived from endoplasmic reticulum (desmotubule) and the cell wall surrounding the plasma membrane ${ }^{19,20}$. The conductivity of the sieve tubes can be regulated via callose ( $\beta-1,3-$ glucan) deposition at the neck region of plasmodesmata in the sieve plates ${ }^{21}$. Callose has been shown to be involved in the regulation of the sieve pore diameter. In cals 7 mutants reduced callose deposition at the sieve plate leads to a reduced number of open pores per sieve plate, smaller diameter sieve pores and impaired phloem transport ${ }^{22,23}$. But also over accumulation of callose, as shown by the phloem-specific expression of a gain of function mutant of CalS3, inhibits phloem conductivity ${ }^{24}$, suggesting that callose deposition at the sieve plate has to be tightly regulated. The only gene identified so far which is specifically required to establish phloem identity is altered phloem development (APL), a MYB coiled-coil-type transcription factor $^{25}$. Despite its importance for plant growth, the mechanisms regulating phloem development and especially sieve plate formation is still poorly understood.

In Arabidopsis, seven genes share sequence similarity to CTL genes in animals and yeast. Here, we show that one of the Arabidopsis CTL homologues, At3g15380, named here as CHER1, mediates choline transport and loss-of-function results in an altered choline metabolite profile, defects in sieve plate and sieve pore formation and impaired phloem transport. CHER1 is expressed in several tissues, localizes to the trans-Golgi network (TGN) and transiently to the forming cell plate during cytokinesis. In addition, during early SE differentiation, CHER1 has a polar localization associated with the forming sieve plates. Together, our results indicate that a CTL-like protein functions in a choline transport process that is involved in sieve plate and sieve pore formation in plants.

\section{Results}

Isolation of cher1 mutants with altered AHP6 expression. To identify novel regulators of vascular patterning and transport, we carried out a misexpression screen for pAHP6::GFPer, a cytokinin signalling inhibitor. pAHP6::GFPer is normally expressed in a highly specific pattern associated with xylem ${ }^{26}$ (Fig. 1d), and its expression pattern is known to respond to the transport status of the phloem ${ }^{27}$. Screening ethyl methanesulfonate (EMS) mutagenized plants expressing pAHP6::GFPer resulted in the isolation of three mutants (Fig. 1c) with similar variable, often expanded $p A H P 6::$ GFPer expression $(50 \%, n=20$; Fig. 1e), which we further characterized. An allelism test revealed that the three isolated mutants are allelic.

cher1 mutants exhibit defects in root development. All three cher1 mutant alleles are dwarf with defects in both shoot and root architecture (Fig. 1c). Shortly after germination, cher1 mutants develop a severe root growth phenotype that involves reduced primary root growth and lateral roots that grow approximately the same length as the primary root. Six-day-old cher1 seedlings develop a primary root about $10 \%$ of the length of a wild-type (WT) root (Supplementary Fig. 1a). Also the size of the root meristem region decreased during the first 5-6 dag as determined by propidium iodide-stained roots using confocal laser scanning microscope (Supplementary Fig. 1b,c). Severe swelling of ground tissue cells, abnormal root hair development and aberrant cell wall positioning are further characteristics of cher1-1 mutant (Supplementary Fig. 1d,e). Consistent with misexpression of pAHP6::GFPer, altered xylem specification was detected in the stele of cher1 mutants. Fuchsin staining of cher1 mutants revealed that the distinct patterning of meta- and protoxylem as observed in WT roots is abolished. The xylem in cher1-1 mutant is often discontinuous, some of the roots developed extra meta- or protoxylem files (Supplementary Fig. 1f,g).

CHER1 is required for phloem patterning and conductivity. To follow-up the pAHP6::GFPer misexpression aspect, which is informative for phloem function, we next analysed vascular conductivity in the cher1-1 mutant. In WT plants, green fluorescent protein (GFP) expressed under control of the CC-specific pSUC2 promoter $(p S U C 2:: G F P)^{28}$ is translocated from CC to SEs and unloaded symplastically into the root meristem (Fig. 1f,h). In cher1-1 mutant, the unloading of GFP from protophloem into the root meristem is blocked (Fig. 1g,i). Reduced symplastic movement was further observed in cher1-1 mutants expressing pSUC2::SpoGFP ${ }^{29}$, a Sporamin fused to GFP to increase the 
a

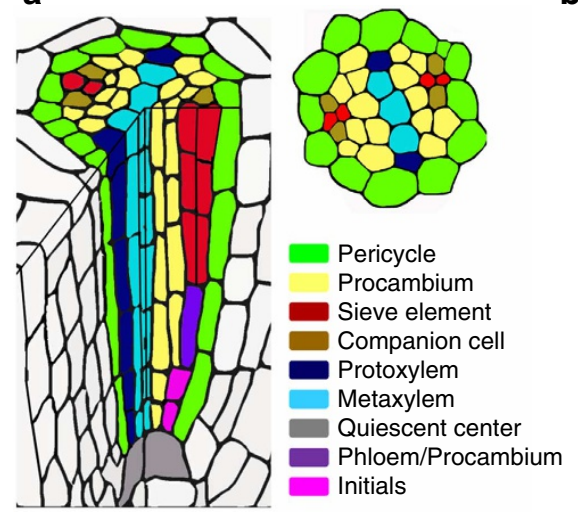

b

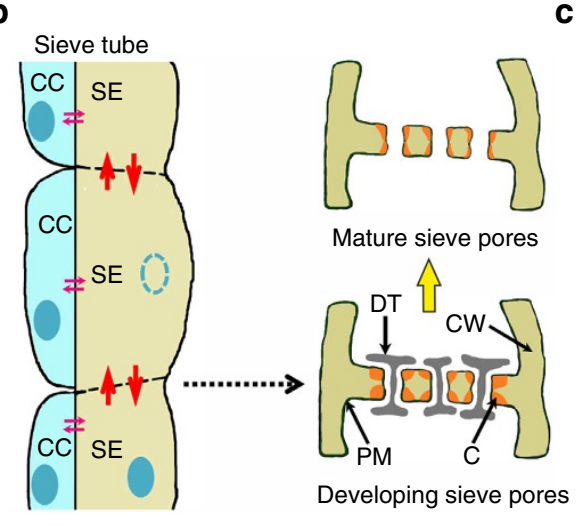

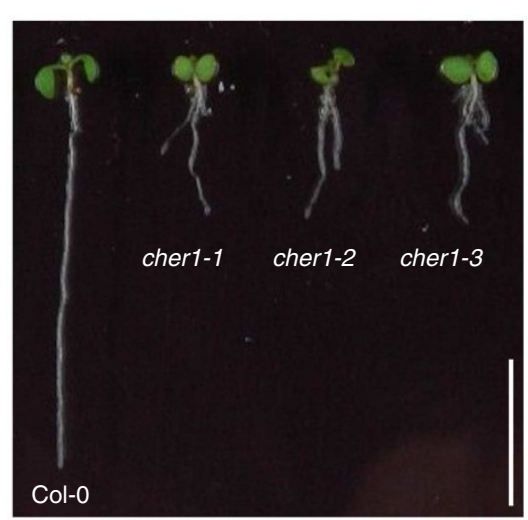
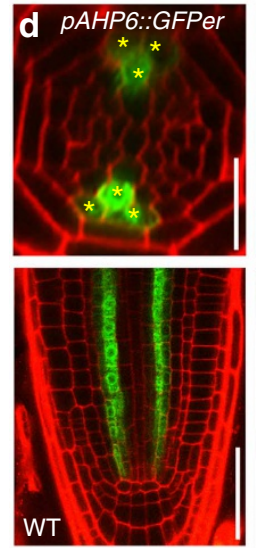
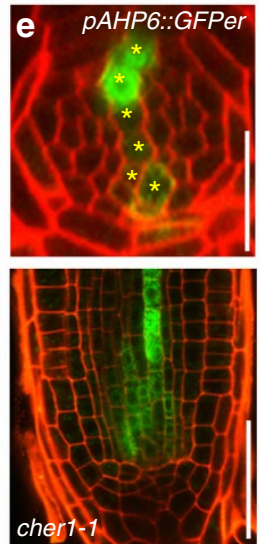
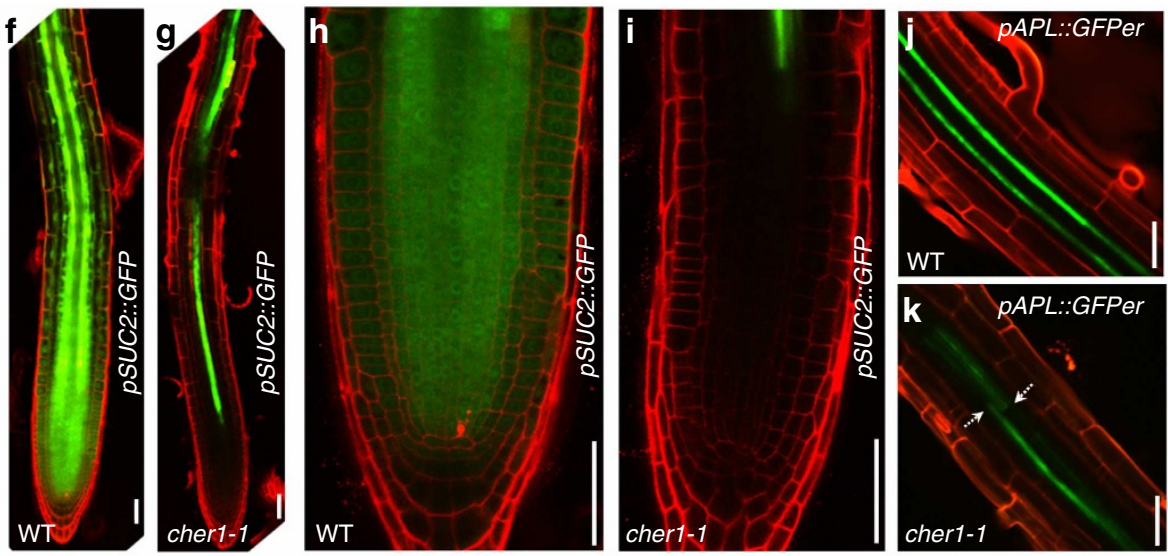

Figure 1 | cher1-1 mutants show altered pAHP6:-GFPer expression and abnormal phloem development. (a) Schematic presentation of the Arabidopsis root highlighting the vascular cylinder. (b) Schematic of sieve tube structure and sieve pore maturation. (c) Seedling phenotype of 6-day-old wild-type (Col-0), cher1-1, cher1-2 and cher1-3. Scale bar, $1 \mathrm{~cm}$. (d,e) pAHP6::GFPer in wild-type Col-0 (d) and cher1-1 (e). Pericycle and protoxylem cells expressing pAHP6::GFPer are marked with yellow asterisks. Scale bars represent $50 \mu \mathrm{m}$ for longitudinal and $25 \mu \mathrm{m}$ for cross-sections. (f-i) pSUC2::GFP in wild-type Col$0(\mathbf{f}, \mathbf{h})$ and cher1-1 (g,i) (the tip region is shown at higher magnification in $\mathbf{h}$ and $\mathbf{i})$. (j,k) pAPL::GFPer in wild-type (j) and cher1-1 (k). White arrows indicate the sites of fragmented PAPL::GFPer expression. Scale bar, $50 \mu \mathrm{m}$.

protein size (Supplementary Fig. 1h-k). These results indicate that the cher1-1 mutant is defective in symplastic transport through phloem.

To understand the status of phloem conductivity, we next investigated phloem development in the cher1-1 mutants. Toluidine blue-stained cross-sections of 6-day-old cher1-1 roots revealed severe defects in procambium maintenance and phloem patterning. WT phloem poles consist of two CCs and two SEs (Supplementary Fig. 11), whereas cher1-1 mutants exhibit an increased number of SE-like cells frequently extending into the procambial domain (Supplementary Fig. 1m). Furthermore, we observed a disrupted pattern of the phloem-specific $p A P L:: G F P e r$ confirming that continuous phloem cell differentiation is unstable in cher1-1 mutant (Fig. 1j,k).

CHER1 encodes a plant CHOLINE TRANSPORTER-LIKE protein. To understand the role of the CHER1 gene product in regulating phloem morphogenesis or phloem conductivity, we next set out to better characterize the mutant locus. Rough mapping via a PCR-based positional cloning approach indicated the locus was between the markers J3-5079754 and J3-5255497 leaving a window of approximately $175 \mathrm{~kb}$. Subsequent wholegenome re-sequencing (Illumina) revealed a premature STOP in the CHOLINE TRANSPORTER-LIKE (AtCTL1, At3g15380) (30,31 $^{3}$ gene, which we rename here as CHER1. Two other cher1 mutant alleles with premature STOP codons were identified from the same forward genetic screen. All three cher1 alleles (cher1-1 (TGG $\rightarrow$ TAG at position 1403 of CDS), cher $1-2$ (TGG $\rightarrow$ TGA at position 438 of CDS) and cher1-3 (CGA $\rightarrow$ TGA at position 946 of CDS) ) and a T-DNA insertion mutant (SALK_065853; Fig. 2a) segregate in a recessive manner. In addition, we were able to complement cher1-1 with a 3117-bp genomic DNA encompassing the CHER1 coding sequence and the $1,337 \mathrm{bp}$ $5^{\prime}$-upstream region (Fig. 2b). The CHER1/At3g15380 locus encodes a protein of 700 amino acids, with ten predicted transmembrane spanning domains (TMHMM Server v. 2.0) similar to the predictions of most CTL proteins from rat or human and other organisms ${ }^{3}$. In plants, seven genes including CHER1/ At3g15380 have been annotated as potential CTL proteins in Arabidopsis based on their protein sequence similarity (Supplementary Fig. 2a). However, our in sillico analysis with the multiple alignment with CTL proteins from various organisms revealed that CHER1 is closest in homology to the animal CTL proteins and is distinct from the other six Arabidopsis CTL-like proteins (Supplementary Fig. 2b,c).

CHER1 facilitates choline transport. The first CTL protein was isolated by screening a cDNA library from the Torpedo lobe, which is highly enriched in neurons to identify genes able to suppress the growth defects of a yeast mutant lacking choline transport and reduced choline metabolism ${ }^{9,32}$. Choline uptake experiments performed with CTL1 and CTL2 from different 


\section{a}

CHER1, At3g15380

CHER1, At3g15380

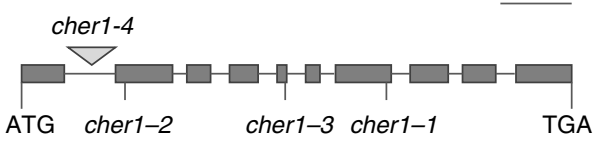

cher1-1 TGG > TAG

cher1-2 TGG > TGA

cher1-3 CGA > TGA

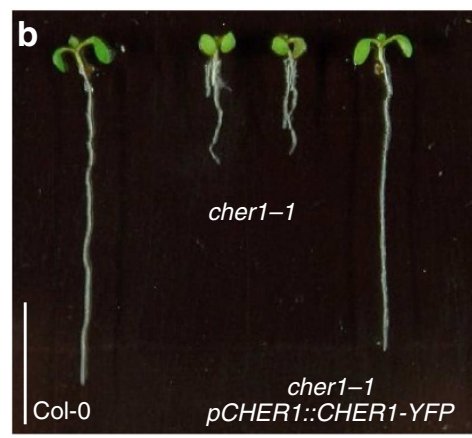

d

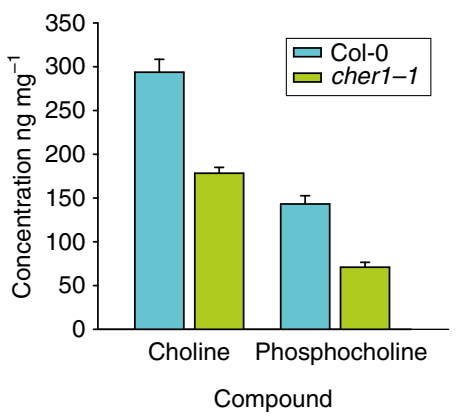

$\mathbf{f}$

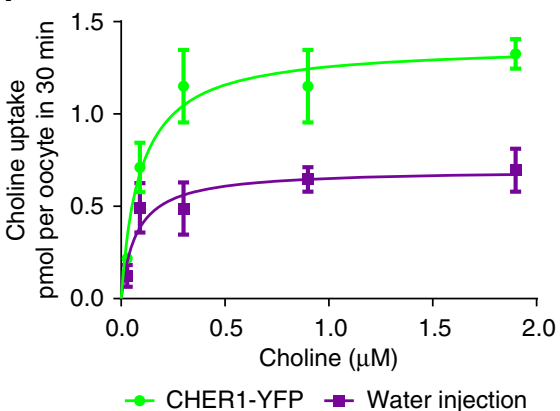

Figure 2 | CHER1 facilitates choline transport. (a) Schematic view of the intron-exon structure of CHER1 and sites of cher1-1, cher1-2, cher1-3 mutations and T-DNA insertion cher1-4 (SALK_065853). (b) Seedling phenotype of 6-day-old wild-type Col-0, cher1-1 and cher1-1 rescued with pCHER1::CHER1-YFP. Scale bar, $1 \mathrm{~cm}$. (c) Choline, glucose and auxin (IAA) uptake by Xenopus oocytes expressing CHER1 cDNA. Auxin has been selected as a negative control and has been tested previously in oocytes ${ }^{69}$. Error bars $=$ s.e.m. $\left(n=6\right.$ independent experiments, $P=0.0052$ for $10 \mu \mathrm{M}{ }^{14} \mathrm{C}$-choline by Student's $t$-test using GraphPad Prism 5). (d) Choline and phosphocholine levels in Col-0 and cher1-1 roots. Error bars =s.d., three biological replicates were used for cher1-1 and four biological replicates for wild type. (e) Time-dependent choline uptake at $0.3 \mu \mathrm{M}{ }^{14} \mathrm{C}$-choline by CHER1-YFP-expressing and water-injected oocytes. After subtraction of the background, the linear regression indicated a twofold difference in the slope between water-injected and CHER1-YFP-injected oocytes. Representative of four separate experiments at low choline concentrations (0.3-1 $\mu \mathrm{M}$ ), error bars represent the s.e.m. of four to six oocytes. (f) A kinetic analysis of choline transport shows that CHER1 expression increases the $V_{\max }$ from 0.70 to $1.37 \mathrm{pmol} / 30$ min without changing the $K_{\mathrm{m}}$ ( 0.07 versus $0.09 \mu \mathrm{M})$. Good fits $\left(R^{2}=0.96\right.$ for CHER-YFP- and 0.88 for water-injected oocytes, 24 oocytes for each condition, nonlinear fitting was done using GraphPad Prism 5) were obtained with a simple Michaelis-Menten equation after subtraction of the baseline measured in the presence of $10^{4} \mathrm{M}$ unlabelled choline. Representative of three separate experiments.

organisms using various heterologous cell systems showed that these CTL proteins can facilitate choline transport across membranes ${ }^{9,32-36}$. To test whether CHER1 also has the capability to transport choline, uptake experiments were performed using Xenopus oocytes. Expression of CHER1-GFP fusion proteins in the oocytes revealed that the tagged protein localizes primarily to intracellular compartments. However, the high levels of exogenous protein produced in the oocytes allow a small portion to be localized in the plasma membrane because of cell membrane trafficking, and the expression of in vitro transcribed tagged CHER1 RNA was able to increase choline uptake 1.3-fold (Fig. 2c) in the oocytes, which is similar to the uptake rates observed with tagged human CTL1 (Supplementary
Fig. 3a). Both endogenous and CHER1-related choline uptake were time (Fig. 2e and Supplementary Fig. 3a) and temperature (Supplementary Fig. 3b) dependent. The time-dependent uptake at $0.3 \mu \mathrm{M} 14 \mathrm{C}$-choline revealed a twofold difference in the slope between water-injected and CHER1-YFP-injected oocytes (Fig. 2e). Moreover, the transport kinetics analysis showed that CHER1 increased the Vmax of a high affinity component of choline transport in the oocytes (Fig. 2f). A similar effect on Vmax has been observed previously with rat rCTL1a and rCTL1b proteins $^{36}$. We observed a stimulatory effect of low $\mathrm{pH}$ on choline uptake in the presence of CHER1 (Supplementary Fig. 3c). In contrast, the transport of indole-3-acetic acid (IAA) and glucose by oocytes was not affected by the expression of CHER1 
(Fig. 2c). To determine whether the loss of function mutations in CHER1 cause a reduction in choline and phosphocholine levels, we measured the total content of these molecules in the root of 7 -day-old seedlings. The quantification revealed a significant reduction of Cho (40\%, $P<0.0001$ Student's $t$-test) and PCho (51\%, $P<0.0001$, Student's $t$-test) content in cher $1-1$ compared with that present in WT plants (Fig. 2d), indicating that CHER1 may be involved in the regulation and/or maintenance of choline metabolite levels in plants. Furthermore, we analysed the expression of $p A P L:: G F P e r$ and the distribution of the freely moving GFP driven by the phloem CC-specific $p S U C 2$ promoter during phloem development in the biosynthetic xipotl mutant, which also shows reduced levels of choline and phosphocholine ${ }^{16,37}$. Similar to cher1-1, we observed a reduced movement and unloading defects of $p S U C 2:: G F P$ (Supplementary Fig. $4 \mathrm{a}-\mathrm{d}$ ), in addition to the fragmented expression pattern of $p A P L:: G F P e r$ (Supplementary Fig. 4e,f) in xipotl phloem and an increased number of SE-like cells in the root crosssection (Supplementary Fig. 4g,h). Taken together, these data indicate that CHER1 facilitates choline transport and maintains choline homeostasis during root (and phloem) development in Arabidopsis.
CHER1 localizes to the TGN, forming cell- and sieve-plates. We next investigated the subcellular localization of CHER1 in the meristematic cells of Arabidopsis root by expressing a functional pCHER 1::CHER1-YFP in WT and cher1-1 mutants. We found that $p$ CHER 1::CHER1-YFP displayed a punctate pattern in the cytosol in close vicinity to the Golgi marker CD3-967-RFP ${ }^{38}$ and colocalizes with the TGN/EE (early endosome) labelled by VHAa1-RFP (Fig. 3a-c). Transmission electron microscopy of immunogold labelled ultrathin cryosections using GFP antibodies confirmed the presence of pCHER1::CHER1-YFP in the TGN/EE (Fig. 3d). Notably, in the mitotic cells, pCHER1::CHER1-YFP is present at the sides of centrifugally extending cell plates as indicated by the ring-like localization in a three-dimensional (3D) projection (Fig. 3e-h; Supplementary Movie 1). This localization suggests that $p C H E R 1:: C H E R 1-Y F P$ accumulates at the phragmoplast, the site where vesicles are transported to the expanding cell plate. $4^{\prime}, 6$-Diamidino-2phenylindole staining of cortex and epidermis cells expressing pCHER1::CHER1-YFP shows a specific requirement for CHER1 at the cell plate during the telophase stage of cell division (Fig. 3h).
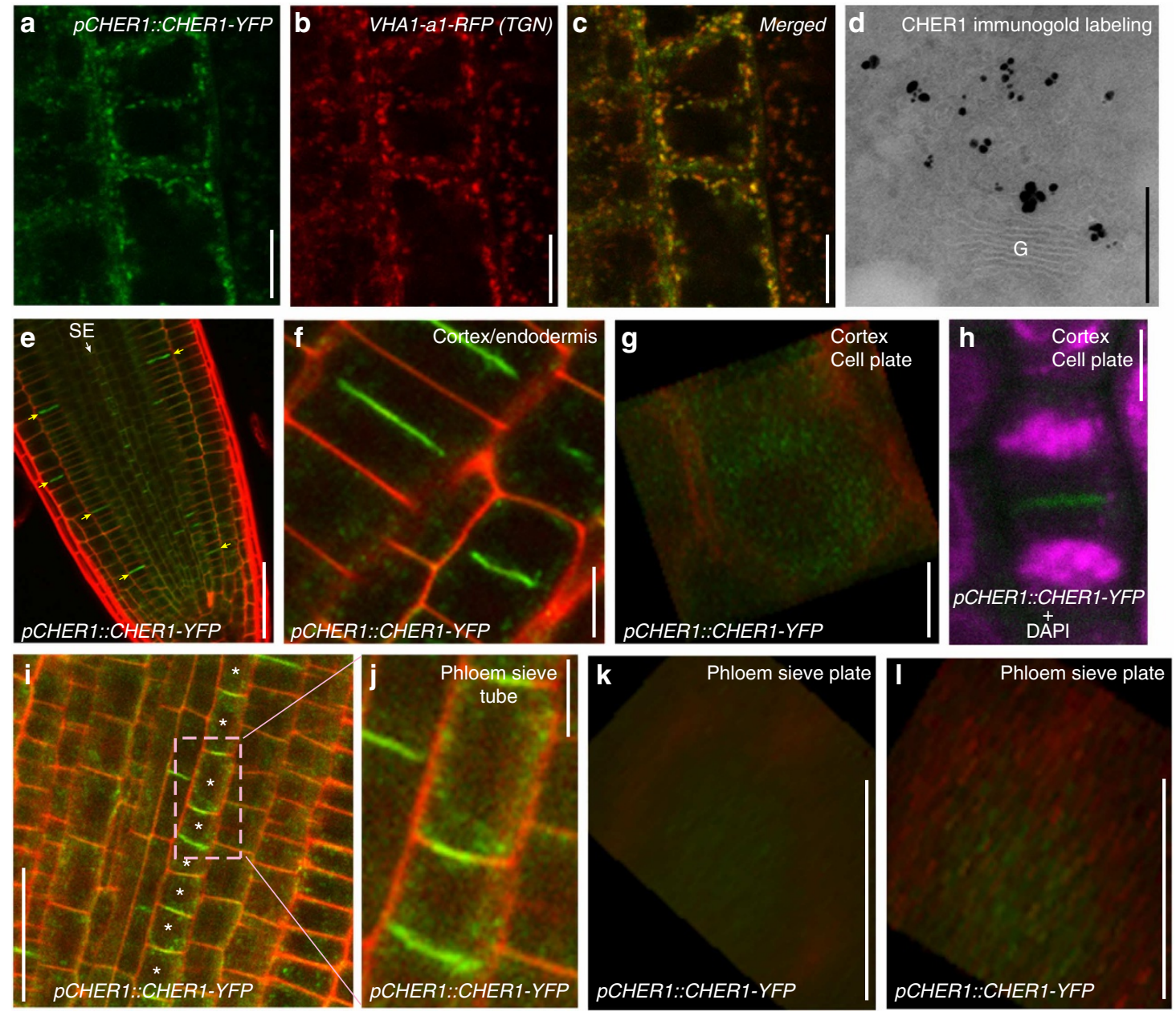

Figure 3 | CHER1 localizes to TGN, nascent cell plates and in phloem precursor cells to the sites of forming sieve plates. (a-c) Colocalization of pCHER1::CHER1-YFP with TGN marker VHA-a1-RFP. Scale bar, $7.5 \mu \mathrm{m}$. (d) Immunogold labelling of CHER1 in Arabidopsis root. G, Golgi. Scale bar, $250 \mathrm{~nm}$. (e) PCHER1::CHER1-YFP expression in phloem and outer layers of the root. Yellow arrows indicate cell plate-specific CHER1 expression in outer layers of the root; white arrow indicates sieve element-specific CHER1 expression. Scale bar, $50 \mu \mathrm{m}$. (f) pCHER1::CHER1-YFP expression in cortex and epidermis cells. Scale bar, $7.5 \mu \mathrm{m}$. (g) pCHER1::CHERT-YFP ring-like localization in a 3D projection at the cell plate of cortex and endodermis cells. Scale bar, $5 \mu \mathrm{m}$. (h) 4',6-Diamidino-2-phenylindole (DAPI) staining of endodermis cell expressing pCHER1::CHER1-YFP. Scale bar, $7.5 \mu \mathrm{m}$. (i,j) Polar CHER1 localization in the early phloem sieve elements. Protophloem sieve elements are marked with asterisks. Scale bar, $25 \mu \mathrm{m}$. (k,I) Sustained expression pattern of CHER1 in sieve plates in a 3D projection. Scale bars, $5 \mu \mathrm{m}$. 
Whereas in most root meristem cells, pCHER1::CHER1-YFP is predominantly found at the TGN or at the phragmoplast in dividing cells, in SE cells $p$ CHER1::CHER1-YFP exhibited a specific subcellular localization, in which CHER1-YFP accumulates in a polar manner at the central domain of the developing sieve plate (Fig. 3e,i-l). Compared with the transient ring-like localization in nascent cell plates (Fig. 3g), CHER1-YFP in the central domain of sieve plates is more sustained (Fig. 3k,l; Supplementary Movie 2). These data suggest that CHER1 has a specific function in the elaboration of the sieve plates/pores.

Continuous vesicle trafficking maintains CHER1 polarity. In (cholinergic) neurons, acetylcholine synthesis is maintained by the high-affinity CHT1, which transports choline across the plasma membrane. By influencing CHT1, endocytosis and trafficking choline uptake and hence acetylcholine biosynthesis can be regulated $^{39}$. To investigate whether continuous membrane trafficking is required to maintain CHER1 polarity in SE cells, we treated seedlings expressing CHER1(cDNA)-YFP under control of an early phloem-specific promoter $p E P M^{40}$ with brefeldin A (BFA). BFA is a fungal toxin that inhibits vesicle transport and involves GDPGTP exchange factors for small G proteins of the ARF class (ARF GEFs). In plants, BFA has been shown to block endocytic recycling of certain proteins and causes an aggregation of endosomes (including TGN) and internalized endocytic cargos into so-called
BFA compartment ${ }^{41}$. In untreated seedlings, we observed pEPM::CHER1(cDNA)-YFP expression at the TGN and a polar pattern in phloem precursor cells (Fig. 4a,b). By contrast, BFA treatment caused rapid intracellular aggregation of CHER1 proteins into BFA compartments and loss of the polar pattern in the early sieve plates (Fig. 4c,d), indicating that continuous vesicle trafficking is required for creating CHER1 polarization in SE. Furthermore, we analysed the behaviour of two dynamin-related proteins, DRP1A and DRP1C, which are involved in the clathrinmediated endocytosis in plants ${ }^{42-44}$. As previously reported, both DRP1A and DRP1C localize to the cell plate and the plasma membrane of expanding and fully expanded interphase cells ${ }^{44,45}$. Notably, we found that both DRP1A and DRP1C exhibit a similar polarized pattern to that of CHER1 in SE cells (Fig. 4e-h). Taken together, our observation here suggests that a BFA-sensitive endocytotic recycling mechanism might be involved in the maintenance of the polar CHER1 pattern in SE cells.

CHER1 is required for sieve plate and sieve pore elaboration. The intriguing polar localization of CHER1 in SE cells suggests that CHER1 may have a role in the elaboration of sieve plates. Using serial block-face scanning electron microscopy (SBEM) ${ }^{46}$, we next compared serial sectioned roots of two WT (from 235 to $562 \mu \mathrm{m}$ and from 210 to $525 \mu \mathrm{m}$ from the root tip) and two cher1-1 (from 162 to $408 \mu \mathrm{m}$ and from 129 to $445 \mu \mathrm{m}$ from the
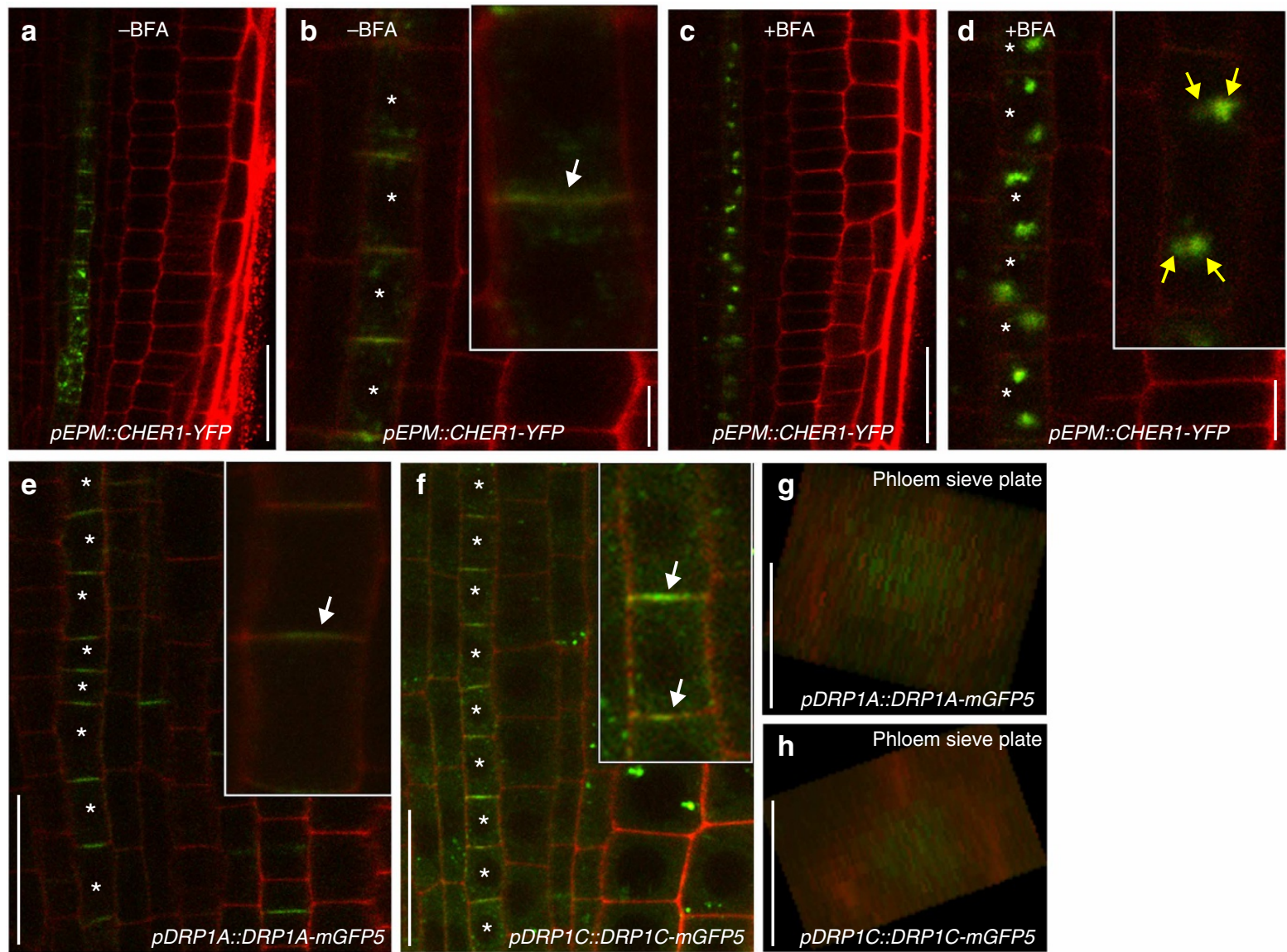

Figure 4 | Continuous vesicle trafficking is required for establishing CHER1 polarization and sustained pattern maintenance in the developing sieve plates. (a,b) pEPM::CHERT-YFP expression in early sieve elements. Asterisks indicate phloem sieve elements and white arrows indicate polar CHER1 localization in the magnified image (b). Scale bars, $25 \mu \mathrm{m}$ for $\mathbf{a}$ and $5 \mu \mathrm{m}$ for $\mathbf{b}$. (c,d) pEPM::CHER1-YFP expression after 30 min of $50 \mu \mathrm{M}$ BFA treatment. Asterisks indicate phloem sieve elements and yellow arrows indicate BFA compartments in the magnified image (d). Scale bars represent $25 \mu \mathrm{m}$ (c) and $5 \mu \mathrm{m}$ (d). (e) pDRP1A:DRP1A-mGFP5 expression in the early sieve elements. (f) pDRP1C:DRP1C-mGFP5 expression in the early sieve elements. Asterisks indicate phloem sieve elements and white arrows indicate polar DRP1A and DRP1C expression pattern. (g,h) Sustained expression pattern of DRP1A (g) and DRP1C (h) in sieve plates in a 3D projection. Scale bars, $5 \mu \mathrm{m}$. 
a

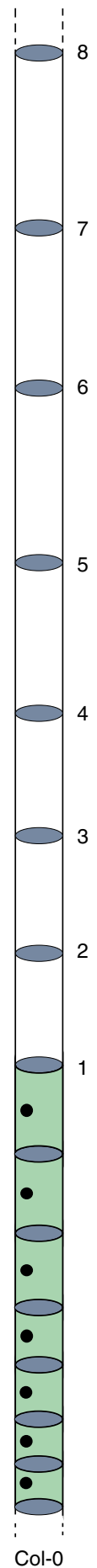

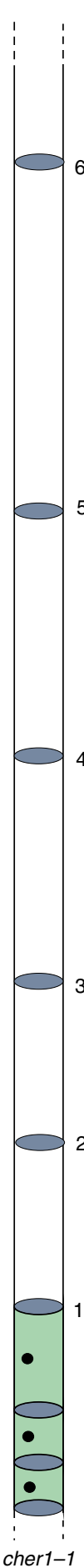

b

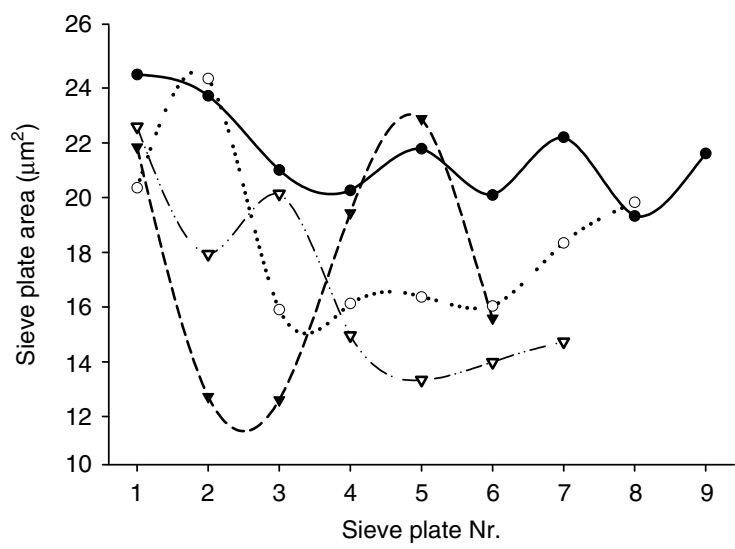

C

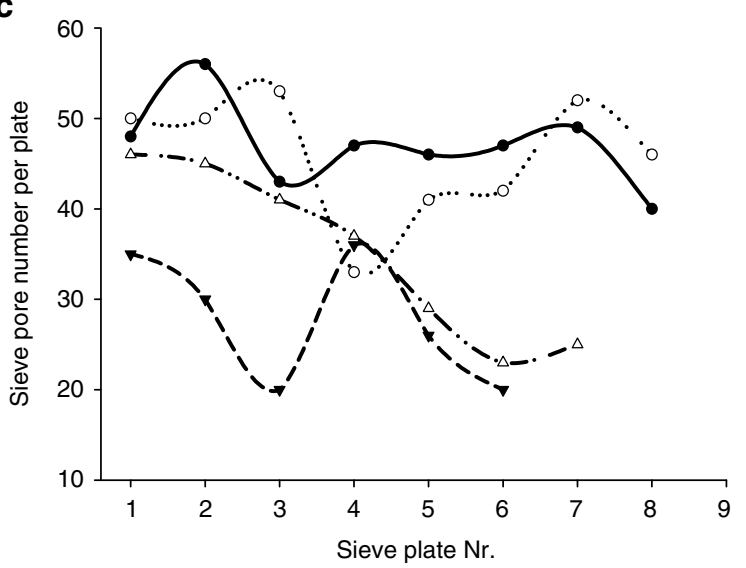

d

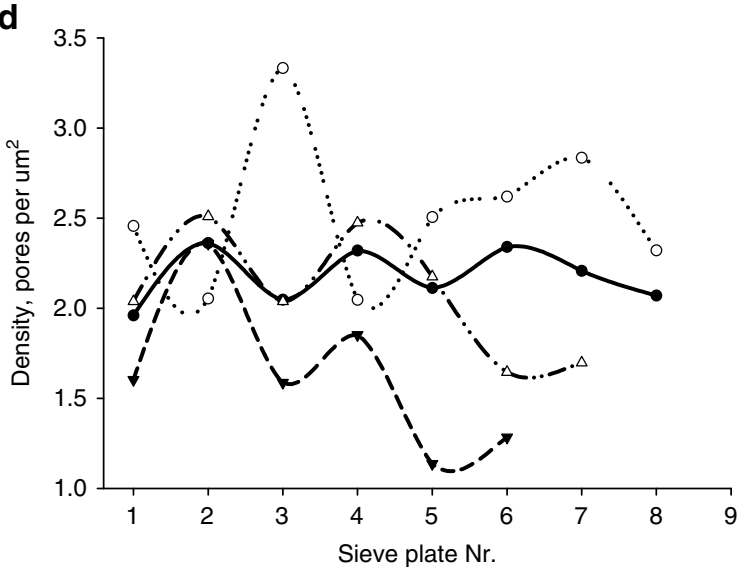

$\longrightarrow$ Col-0 sample 1 - - ---- cher1-1 sample 1

........ Col-0 sample 2 _. - .... cher1-1 sample 2

Figure 5 | Sieve pore density is reduced in cher1 mutant. (a) Schematic view of developing Col-0 and cher1-1 sieve elements. Sieve elements in cher1 mutant become elongated earlier than in Col-0 roots. Sieve plates taken for analysis are numbered accordingly. (b-d) Analysis of sieve pore area (b), sieve pore number (c) and sieve pore density (d) in two independent wild-type Col-0 and two cher1-1 roots. The plate between 'green' and 'white' cells is taken as a starting point for analysis.

root tip) seedling root tips from the quiescent center (QC) to the region in the root where enucleated SEs start to be formed (Fig. 5a). In addition, we analysed the sieve plates in mature cher1-1 and WT roots (Fig. 6c,d). The data revealed that, despite having a reduced sieve plate area $\left(17.16 \mu \mathrm{m}^{2}\right.$ in cher1-1 and $20.01 \mu \mathrm{m}^{2}$ in WT average), cher1-1 mutants have a reduced sieve pore density (1.86 pores per $\mu \mathrm{m}^{2}$ in cher $1-1$ and 2.35 pores per $\mu \mathrm{m}^{2}$ in WT average) when compared with WT (Fig. 5b-d). As sieve pores are necessary for the translocation of various microand macromolecules from one phloem cell to the next and thereby enable long-distance transport throughout the plant body, having a reduced sieve pore number in cher1-1 is likely to explain the impaired transport of GFP in the phloem observed with proSUC2::GFP mentioned above.

As the SE differentiates, it undergoes a selective breakdown and all the remaining components become distributed along the 
wall ${ }^{47,48}$. Studies of SE formation in various plant species revealed that this process is accompanied by plasmodesmata enlargement and disintegration of the desmotubules, which leads to the formation of mature sieve plate pores ${ }^{49,50}$ (Fig. 1b). Callose deposition around plasmodesmata has been suggested to have an important role in initiating this process ${ }^{23}$.

Using the SBEM, we observed that during the differentiation of SEs the sieve pores are gradually losing the desmotubules, rigid structures derived from endoplasmic reticulum that run between two SEs. For our analysis, we selected the sieve plates at the junction between 'grey' (cell which still undergoes the selective breakdown) and 'white' (lacking most of cellular compartments) cells and additionally the sieve plates between already elongated and differentiated SEs (Supplementary Fig. 5a). We could not detect a significant difference in the sieve pore anatomy in the junction between 'grey' and 'white' cells in WT $(361 \mu \mathrm{m}$ from the root tip; left model in Fig. 6e and Supplementary Fig. 5b,c) and the cher1-1 mutant $(226 \mu \mathrm{m}$ from the root tip; left model in Fig. $6 \mathrm{f}$ and Supplementary Fig. 5b), where the sieve pores still retained the desmotubules. However, the analysis of the sieve pores in already developed SEs revealed a significant difference between WT and cher1-1. The majority of sieve pores in the WT $(468 \mu \mathrm{m}$ from the root tip; right model in Fig. 6e and Supplementary Fig. 5b,c) have lost the desmotubules, whereas cher $1-1$ sieve pores $(445 \mu \mathrm{m}$ from the root tip) still retained the desmotubules in the symplastic space (right model in Fig. $6 \mathrm{f}$ and Supplementary Fig. 5b,c). Finally, we found that the sieve plate area, sieve pore number and sieve pore area are reduced in 30-day-old xipotl and cher1-1 roots compared with WT (Supplementary Fig. 4i-k). Taken together, our analyses indicate that the function of CHER1 as a facilitator of choline transport is required for the proper sieve pore formation in developing SEs.

\section{Discussion}

In fungi and animals, the shortage of notable $c t l$ mutant phenotypes under non-stress/-induced conditions ${ }^{9,51,52}$ has hampered so far the investigation of CTL-dependent developmental processes. Here we report the identification of CHER1 as a novel regulator of sieve plate formation and provide evidence that CHER1 is involved in maintaining choline homeostasis in the Arabidopsis root.

Enucleated sieve tubes of the phloem form a conductive tissue to distribute various molecules within plants. In order to facilitate long-distance transport, the cell walls separating neighbouring sieve tubes are perforated by sieve pores. Despite its importance for growth and fitness of higher plants, little is known about the genetic regulation of phloem development.

Both expression analysis of phloem marker genes and 3Dscanning electron microscopy (SEM) data revealed that our novel loss-of-function cher 1 mutants fail to unload free (cytosolic) GFP from the phloem into the root tip and form a reduced number and structurally altered sieve pores in sieve plates. As phloem conductance is predicted to be directly linked to the density and size of pores on the sieve plate ${ }^{53,54}$, we propose that CHER1 is required for sieve plate elaboration and hence influences phloem long-distance transport and continuity. This is further supported by the initial observation that the expression domain of AHP6, which is regulated by the function of phloem-derived phytohormone ${ }^{27}$, is expanded in cher 1 mutants. Consistent with its role in phloem development, CHER1 accumulates at the sites of forming sieve plates but it is also localized to the TGN and forming cell plates in other root cell types.

Endocytosis allows cells to adjust the protein composition and localization at the plasma membrane in response to developmental and environmental cues. This process has been shown to regulate the abundance and polarity of various transporters, such as CHT1 in human embryonic kidney and neuroblastoma cells $^{39,55-58}$, aquaporins ${ }^{59}$ and PIN-FORMED (PIN) auxin efflux carriers ${ }^{60-62}$ in Arabidopsis. Our observation that CHER1 is rapidly endocytosed at forming cell plates suggests that a specific vesicle transport machinery is employed to continuously target CHER1 to the forming sieve plates.

Consistent with data from transport assays performed with CTLs from various organisms $9,11,35$, expression of CHER1 in Xenopus oocytes increased choline uptake from the medium. As
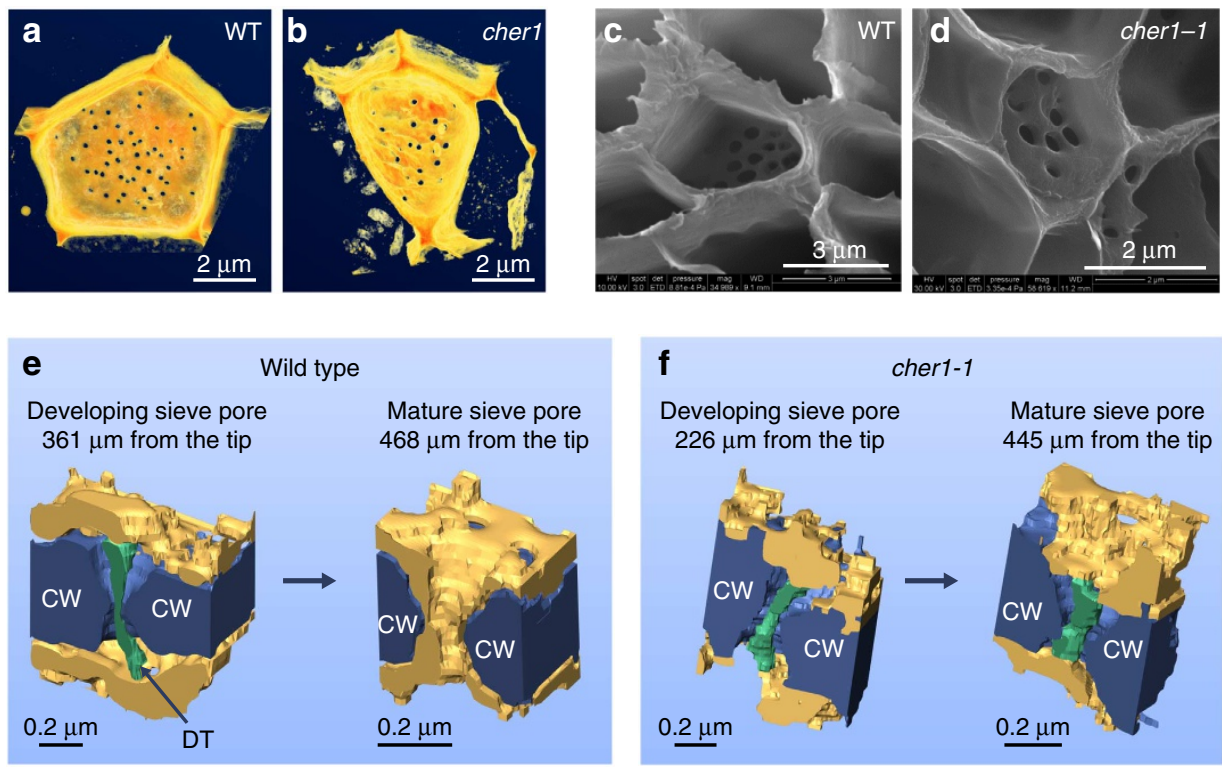

Figure 6 | CHER1 is required for sieve plate and sieve pore formation. (a,b) Reconstructed SBF-SEM images of wild-type Col-0 (at $377 \mu$ m; a) and cher1- 1 (at $310 \mu \mathrm{m} ; \mathbf{b}$ ) sieve plates. Scale bars, $2 \mu \mathrm{m}$. (c,d) SEM images of wild-type Col-0 (c) and cher1-1 sieve plates (d). (e,f) Models of developing and mature sieve pores. The following sieve pores were selected to model 3D structure: developing and mature sieve pores in Col-0 (361 and $468 \mu \mathrm{m}$ from the root tip correspondingly; e), developing and mature sieve pores in cher1-1 (226 and $445 \mu \mathrm{m}$ from the root tip correspondingly; f). CW, cell wall; DT, desmotubule. Scale bars, $0.2 \mu \mathrm{m}$. 
the loss of CHER1 results in reduced choline metabolite levels in the root, it is likely that CHER1-mediated maintenance of choline levels is crucial for proper root development. This notion is further supported by the phenotypic similarity of cherl and the phosphocholine biosynthetic xipotl (xpl) mutant ${ }^{16}$.

At this stage, our work does not fully reveal the cellular process for which CHER1 is required for sieve plate development. Further investigations will be required to clarify the molecular and physiological function of CTLs in plants. For this analysis, the three cher1 alleles of Arabidopsis may provide an important resource.

\section{Methods}

Plant materials and growth conditions. WT Columbia (Col-0) mutants cher1-1, cher1-2 and cher1-3 were isolated in a genetic screen based on EMS mutagenesis of the mobile phloem marker pAHP6::GFPer. The cher1-1 (Col-0) was crossed to Landsberg (Ler) to generate F2 mapping population. The information on mapping, sequencing and genotyping primers is provided in Supplementary Tables. cher1-4 (SALK_065853) was obtained from the Nottingham Arabidopsis Stock Centre. The reporter lines $p A H P 6:: G_{F P e r}{ }^{26}, p A P L:: G_{F P e r}{ }^{25}, p S U C 2:: G_{F P}{ }^{28}$, pSUC2-spoGFP ${ }^{29}$, VHA-a1-GFP ${ }^{63}$ and $P D R P 1 A: D R P 1 A-m G F P 5$ (ref. 45) have been described previously. Seedlings were grown vertically in Petri dishes on $1 \%$ agar, one-half of Murashige and Skoog (MS) basal salt mixture, $1 \%$ sucrose and $0.05 \%$ 4-morpholine ethanesulfonic acid (MES).

Whole-genome re-sequencing. Five microgram of purified genomic DNA in total volume of 50 and $700 \mu \mathrm{l}$ nebulization buffer were added to a nebulizer. Shearing was performed for $6 \mathrm{~min}$ at $32-35$ p.s.i. The recovered volume was $410 \mu$ l. The sample solution was purified and concentrated by column (Qiagen). End repair of the sheared fragments, addition of an A residue to the $3^{\prime}$ end of blunted fragments, and ligation of paired-end (PE) adaptors were according to the Illumina's instructions. The entire adaptor-modified DNA was resolved on a $2 \%$ agarose gel (including $400 \mathrm{ng} \mathrm{ml}^{-1}$ ethidium bromide) run in $1 \times$ TAE buffer (Bio-Rad) for $90 \mathrm{~min}$ at $120 \mathrm{~V}$. Fragments of 300 b.p.s. were excised under illumination from a Dark Reader (Clare Chemical Research). The DNA was then isolated with a Gel Extraction Kit (Qiagen) and $1 \mu \mathrm{l}(2.5 \mathrm{ng})$ was amplified by PCR for 12 cycles with the supplied Illumina PCR primers 1.1 and 1.2 (Illumina). PCR fragments were purified by PCR purification kit (Qiagen) and eluted with $50 \mu \mathrm{l}$ elution buffer (Qiagen)

The library yield was quantified by Nanodrop and diluted to $10 \mathrm{nM}$ in EB buffer (Qiagen) supplemented with $0.1 \%$ Tween-20 for long-term storage at $-20^{\circ} \mathrm{C}$. The DNA sample was sequenced by the Max Planck Institute for Developmental Biology, Tübingen, Germany, on an Illumina GAIIx instrument (Illumina). Wholegenome sequencing data were filtered and trimmed based on per based quality information using $\mathrm{SHORE}^{30}$. Quality controlled data were aligned against the $A$ thaliana reference sequence using GenomeMapper allowing for at most $10 \%$ of the read length to be involved in either gaps or mismatches ${ }^{31,64}$. Consensus calls were performed by SHORE consensus using default parameters. All confident mismatches within the 175-kb mapping interval were annotated according to their effect of gene integrity. The whole-genome sequencing data of cher1-1 have been deposited to the European Nucleotide Archive under accession code PRJEB6417.

Transgenic work. $p A P L:: G F P e r, p A H P 6:: G F P e r, p S U C 2:: G F P$ and $p S U C 2-s p o G F P$ markers were introduced into cher1-1 mutant background by crossing and analyses were done on segregating F2 generations. Transgenic constructs were generated using the Multisite Gateway System (Invitrogen). A 3.1-kb genomic CHER1 fragment was amplified by PCR using primers $5^{\prime}$-AAAAAGCAGGCTATGAGAGGA CCTTTAGGAGCAGTG- $3^{\prime}$ (forward) and $5^{\prime}$-AGAAAGCTGGGTTGTGAGTA AGACTCTGAACCTCTTCC- $3^{\prime}$ (reverse) and cloned into pDONR(Zeo) (Invitrogen) vector and verified by sequencing. Subsequently, the $1.3-\mathrm{kb}$ sequence corresponding to the CHER1 promoter ( $P C H E R 1$ ) was amplified from Col-0 genomic DNA using primers $5^{\prime}$-ATAGAAAAGTTGCTACAAAACTAAGTC GTGCTCTGG-3' (forward) and 5'-TTGTACAAACTTGACTTTGCCACAGCTA TGTCTCTTCAC- $3^{\prime}$ (reverse) and cloned into pDONRP4-P1R (Invitrogen). pCHER1, CHER1 and YFP were combined into the multisite destination vector $\mathrm{pBm} 43 \mathrm{gw}^{65}$ and transformed by floral dipping ${ }^{66}$ into Col- 0 and cher1-1 mutant. To produce $p E P M:: C H E R 1(c D N A)-Y F P$, a 2.1-kb fragment was amplified from Col-0 cDNA by PCR using primers 5'-AAAAAGCAGGCTATGAGAGGACCTTTA GGAGCAGTG-3' (forward) and $5^{\prime}$-AGAAAGCTGGGTTGTGAGTAAGACT CTGAACCTCTTCC-3' (reverse), cloned into $\mathrm{pDONR}(\mathrm{Zeo})$ vector and verified by sequencing. $p E P M^{67}, C H E R 1$ (cDNA) and YFP were combined into the multisite destination vector $\mathrm{pBm} 43 \mathrm{GW}$ and transformed by floral dipping into Col- 0 .

Anatomical analyses and confocal microscopy. For serial sectioning, 5-day-old seedlings were incubated in a fixation solution ( $1 \mathrm{ml}$ of $25 \%$ glutaraldehyde, $2.7 \mathrm{ml}$ of $37 \%$ formaldehyde, $2.5 \mathrm{ml}$ of $\mathrm{NaPi}$ and $18.8 \mathrm{ml}$ of $\mathrm{H} 2 \mathrm{O}$ ) at $4{ }^{\circ} \mathrm{C}$ overnight. After the fixation, the plants were dehydrated in an ethanol gradient $(10 \%, 30 \%, 50 \%$, $70 \%, 96 \%, 2 \times$ absolute ethanol), for at least $30 \mathrm{~min}$ at each step. Next, the seedlings were incubated in a 1:1 mix of absolute ethanol and solution A $(100 \mathrm{ml}$ of Basic resin, $1 \mathrm{~g}$ of Historesin activator (both from Leica Historesin embedding kit) and $2 \mathrm{ml}$ of polyethylene glycol) overnight at room temperature. Consequently, the incubation solution was replaced with pure solution $\mathrm{A}$ and incubated for $2 \mathrm{~h}$ more at room temperature. For embedding, the roots were oriented horizontally next to each other in a plastic well. The well was next filled with a mix of solution A and Historesin hardener (15:1) and covered with a plastic and left for polymerization to take place. As many wells were produced separately, they were finally combined into one block and filled with the previously mentioned polymerization mix. Finally, the resin blocks with embedded roots were attached to the wooden blocks for sectioning. 3-5 $\mu \mathrm{m}$ sections were made on a Leica JUNG RM2055 microtome, using a Leica microtome knife (Leica disposable blades TC-65; Leica). Sections were stained in a $0.05 \%$ toluidine blue solution and visualized with a Leica 2500 Microscope (Leica)

For fuchsin staining, seedlings were first cleared with acidified methanol $(10 \mathrm{ml}$ of methanol, $2 \mathrm{ml}$ of concentrated $\mathrm{HCl}(37 \%)$ and $38 \mathrm{ml}^{\circ} \mathrm{H}_{2} \mathrm{O}$ ) and incubated at $55^{\circ}-57^{\circ} \mathrm{C}$ for $15 \mathrm{~min}$. The acidified methanol was replaced with basic solution $(7 \%$ $\mathrm{NaOH}$ in $60 \%$ ethanol) and incubated for $15 \mathrm{~min}$ at room temperature. The rehydration of the seedlings took place in several steps (40,20 and 10\% ethanol, $10 \mathrm{~min}$ each). The seedlings were stained for $5 \mathrm{~min}$ in $0.01 \%$ basic fuchsin solution destained in $70 \%$ ethanol for 10 min and rehydrated again in the ethanol gradient (40, 20 and 10\%). An equal amount of 50\% glycerol was added to the $10 \%$ ethanol and the seedlings were incubated overnight. Finally, the seedlings were mounted in $50 \%$ glycerol on the glass slides. The images of various markers and fuchsin-stained roots were taken on Leica SP5 confocal microscope using a solid-state blue laser for GFP $(480 \mathrm{~nm} / 270 \mathrm{~mW})$

CHER1, DRP1A and DRP1C localization were examined by confocal $z$-sectioning and $3 \mathrm{D}$ reconstruction. To inhibit the endosomal recycling, the roots were treated with $50 \mu \mathrm{M}$ BFA (Invitrogen).

Expression in Xenopus oocytes and choline uptake assays. Xenopus laevis oocytes were prepared by excision and defolliculation using collagenase D (20 $\mathrm{mg} \mathrm{ml}^{-1}$, Roche Diagnostics), then injected with CHER1 RNA made with mMessage mMachine (Ambion) from the oocyte expression vector pOO2. Uptake measurements were performed after 3-4 days of incubation in Barth's medium. Oocytes were incubated at room temperature with ${ }^{14} \mathrm{C}$-choline $\left(2.04 \mathrm{GBq} \mathrm{mmol}^{-1}\right.$ Perkin-Elmer) at $0.1-10 \mu \mathrm{M}$ for the indicated times, then washed and solubilized for determination of the accumulated radioactivity.

Immunogold labelling. For immunogold labelling of ultrathin thawed cryosections, root tips were fixed with $4 \%(30 \mathrm{~min})$ and $8 \%$ formaldehyde $(2 \mathrm{~h})$, embedded in gelatin and infiltrated with a mixture of polyvinylpyrrolidone and sucrose. Thawed ultrathin sections were labelled with rabbit anti-GFP antibodies (1:300; Abcam) and goat anti-rabbit IgG coupled to Nanogold (no. 2004; Nanoprobes). After silver enhancement (HQ Silver, 8 min; Nanoprobes), sections were embedded in methyl cellulose.

SBEM. SBEM is a method that is based on a combination of a microtome (3View, Gatan) mounted inside the SEM chamber and a sensitive back-scattered electron detector (Gatan) producing classic transmission electron microscopy-like images. The microtome repeatedly shaves resin-embedded specimen to form a fresh block face that is being imaged. After each cutting and imaging step, the specimen is moved up by desired Z-step, and the cycle is automatically repeated.

The roots were fixed with $2.5 \%$ glutaraldehyde, $2 \%$ formaldehyde in $0.1 \mathrm{M} \mathrm{Na}$ Cacodylate buffer ( $\mathrm{pH}$ 7.4) supplemented with $2 \mathrm{mM} \mathrm{CaCl}_{2}$ for $2-3 \mathrm{~h}$ at room temperature, and prepared using a previously described protocol ${ }^{70}$. Durcupan ACM resin (Fluka, Sigma-Aldrich) was mixed according to the manufacture's recommendations. After standard dehydration steps, samples were embedded in silicone holders filled with $100 \%$ Durcupan and infiltrated for at least $2 \mathrm{~h}$ before polymerization at $60^{\circ} \mathrm{C}$. The roots were trimmed to the desired starting point from the tip using EM Ultracut UC6i ultramicrotome (Leica Mikrosysteme GmbH) and a diamond knife. The excess resin around the root was trimmed away and the specimen was mounted on a 3View pin. Finally, the sides of the specimen were covered with silver paint (Agar Scientific Ltd.) and the whole assembly was platinum-coated using Quorum Q150TS (Quorum Technologies, UK). Images were acquired with a FEG-SEM Quanta 250 (FEI), using a backscattered electron detector (Gatan) with 2.5-kV beam voltage, spot size 3 and pressure between 0.15 to 0.3 Torr. The block faces were cut with $40 \mathrm{~nm}$ increments. The images were first processed and segmented using Microscopy Image Browser, a self-developed programme written under Matlab environment, and further visualized in Amira (FEI Visualization Sciences Group)

Scanning electron microscopy. Arabidopsis tissue for SEM investigations of sieve plates was prepared as described previously ${ }^{68}$. In short, plants were shockfrozen in liquid nitrogen, freeze substituted in ethanol and the cytoplasm was digested in $0.5 \%$ Proteinase $\mathrm{K}$ and $8 \%$ Triton X-100 for 2 weeks. The tissue was washed, freeze dried, sputter coated and observed in a FEI Quanta 200 FEG SEM. 


\section{References}

1. Ueland, P. M. Choline and betaine in health and disease. J. Inherit. Metab. Dis. 34, 3-15 (2011)

2. Lockman, P. R. \& Allen, D. D. The transport of choline. Drug. Dev. Ind. Pharm. 28, 749-771 (2002).

3. Michel, V., Yuan, Z., Ramsubir, S. \& Bakovic, M. Choline transport for phospholipid synthesis. Exp. Biol. Med. (Maywood) 231, 490-504 (2006).

4. Apparsundaram, S., Ferguson, S. M. \& Blakely, R. D. Molecular cloning and characterization of a murine hemicholinium-3-sensitive choline transporter. Biochem. Soc. Trans. 29, 711-716 (2001).

5. Apparsundaram, S., Ferguson, S. M., George, A. L. \& Blakely, R. D. Molecular cloning of a human, hemicholinium-3-sensitive choline transporter. Biochem. Bioph. Res. Commun. 276, 862-867 (2000).

6. Okuda, T. et al. Identification and characterization of the high-affinity choline transporter. Nat. Neurosci. 3, 120-125 (2000).

7. Black, S. A. \& Rylett, R. J. Choline transporter CHT regulation and function in cholinergic neurons. Cent. Nerv. Syst. Agents Med. Chem. 12, 114-121 (2012).

8. Michel, V. \& Bakovic, M. The solute carrier 44A1 is a mitochondrial protein and mediates choline transport. FASEB J. 23, 2749-2758 (2009).

9. O'Regan, S. et al. An electric lobe suppressor for a yeast choline transport mutation belongs to a new family of transporter-like proteins. Proc. Natl Acad. Sci. USA 97, 1835-1840 (2000).

10. Ribeiro, F. M. et al. The 'ins' and 'outs' of the high-affinity choline transporter CHT1. J. Neurochem. 97, 1-12 (2006).

11. Traiffort, E., O’Regan, S. \& Ruat, M. The choline transporter-like family SLC44: properties and roles in human diseases. Mol. Aspects Med. 34, 646-654 (2013).

12. Wille, S. et al. Characterization of CDw92 as a member of the choline transporter-like protein family regulated specifically on dendritic cells. J. Immunol. 167, 5795-5804 (2001).

13. Song, P. et al. Choline transporter-like protein 4 (CTL4) links to non-neuronal acetylcholine synthesis. J. Neurochem. 126, 451-461 (2013).

14. Bligny, R., Foray, M. F., Roby, C. \& Douce, R. Transport and phosphorylation of choline in higher-plant cells - P-31 nuclear magnetic-resonance studies. J. Biol. Chem. 264, 4888-4895 (1989).

15. Gout, E., Bligny, R., Roby, C. \& Douce, R. Transport of Phosphocholine in Higher-Plant Cells - P-31 Nuclear-Magnetic-Resonance Studies. Proc. Natl Acad. Sci. USA 87, 4280-4283 (1990).

16. Cruz-Ramirez, A. et al. The xipot1 mutant of Arabidopsis reveals a critical role for phospholipid metabolism in root system development and epidermal cell integrity. Plant Cell 16, 2020-2034 (2004).

17. Yamada, N. et al. Expression and substrate specificity of betaine/proline transporters suggest a novel choline transport mechanism in sugar beet. J. Plant Physiol. 168, 1609-1616 (2011).

18. Chen, C., Li, S., McKeever, D. R. \& Beattie, G. A. The widespread plantcolonizing bacterial species Pseudomonas syringae detects and exploits an extracellular pool of choline in hosts. Plant J. 75, 891-902 (2013).

19. Gallagher, K. L. \& Benfey, P. N. Not just another hole in the wall: understanding intercellular protein trafficking. Genes Dev. 19, 189-195 (2005).

20. Maule, A. J., Benitez-Alfonso, Y. \& Faulkner, C. Plasmodesmata - membrane tunnels with attitude. Curr. Opin. Plant Biol. 14, 683-690 (2011).

21. Zavaliev, R., Ueki, S., Epel, B. L. \& Citovsky, V. Biology of callose (beta-1,3glucan) turnover at plasmodesmata. Protoplasma 248, 117-130 (2011)

22. Barratt, D. H. et al. Callose synthase GSL7 is necessary for normal phloem transport and inflorescence growth in Arabidopsis. Plant Physiol. 155, 328-341 (2011).

23. Xie, B., Wang, X., Zhu, M., Zhang, Z. \& Hong, Z. CalS7 encodes a callose synthase responsible for callose deposition in the phloem. Plant J. 65, 1-14 (2011).

24. Vaten, A. et al. Callose biosynthesis regulates symplastic trafficking during root development. Dev. Cell. 21, 1144-1155 (2011).

25. Bonke, M., Thitamadee, S., Mahonen, A. P., Hauser, M. T. \& Helariutta, Y. APL regulates vascular tissue identity in Arabidopsis. Nature 426, 181-186 (2003).

26. Mahonen, A. P. et al. Cytokinin signaling and its inhibitor AHP6 regulate cell fate during vascular development. Science 311, 94-98 (2006).

27. Bishopp, A. et al. A mutually inhibitory interaction between auxin and cytokinin specifies vascular pattern in roots. Curr. Biol. 21, 917-926 (2011).

28. Imlau, A., Truernit, E. \& Sauer, N. Cell-to-cell and long-distance trafficking of the green fluorescent protein in the phloem and symplastic unloading of the protein into sink tissues. Plant Cell 11, 309-322 (1999).

29. Stadler, R., Lauterbach, C. \& Sauer, N. Cell-to-cell movement of green fluorescent protein reveals post-phloem transport in the outer integument and identifies symplastic domains in Arabidopsis seeds and embryos. Plant Physiol. 139, 701-712 (2005).

30. Ossowski, S. et al. Sequencing of natural strains of Arabidopsis thaliana with short reads. Genome Res. 18, 2024-2033 (2008).

31. Schneeberger, K. et al. Simultaneous alignment of short reads against multiple genomes. Genome Biol. 10, R98 (2009).
32. O'Regan, S. \& Meunier, F. M. Selection and characterization of the choline transport mutation suppressor from Torpedo electric lobe, CTL1. Neurochem. Res. 28, 551-555 (2003).

33. Fullerton, M. D., Wagner, L., Yuan, Z. \& Bakovic, M. Impaired trafficking of choline transporter-like protein-1 at plasma membrane and inhibition of choline transport in THP-1 monocyte-derived macrophages. Am. J. Physiol. Cell Physiol. 290, C1230-C1238 (2006).

34. Kommareddi, P. K. et al. Isoforms, expression, glycosylation, and tissue distribution of CTL2/SLC44A2. Protein J. 29, 417-426 (2010).

35. Nakamura, T. et al. Involvement of choline transporter-like proteins, CTL1 and CTL2, in glucocorticoid-induced acceleration of phosphatidylcholine synthesis via increased choline uptake. Biol. Pharm. Bull. 33, 691-696 (2010).

36. Traiffort, E., Ruat, M., O’Regan, S. \& Meunier, F. M. Molecular characterization of the family of choline transporter-like proteins and their splice variants. J. Neurochem. 92, 1116-1125 (2005)

37. Alatorre-Cobos, F. et al. Translational regulation of Arabidopsis XIPOTL1 is modulated by phosphocholine levels via the phylogenetically conserved upstream open reading frame 30. J. Exp. Bot. 63, 5203-5221 (2012).

38. Nelson, B. K., Cai, X. \& Nebenfuhr, A. A multicolored set of in vivo organelle markers for co-localization studies in Arabidopsis and other plants. Plant J. 51, 1126-1136 (2007).

39. Ribeiro, F. M. et al. The hemicholinium-3 sensitive high affinity choline transporter is internalized by clathrin-mediated endocytosis and is present in endosomes and synaptic vesicles. J. Neurochem. 87, 136-146 (2003).

40. Bishopp, A. et al. Phloem-transported cytokinin regulates polar auxin transport and maintains vascular pattern in the root meristem. Curr. Biol. 21, 927-932 (2011).

41. Grunewald, W. \& Friml, J. The march of the PINs: developmental plasticity by dynamic polar targeting in plant cells. EMBO J. 29, 2700-2714 (2010).

42. Bednarek, S. Y. \& Backues, S. K. Plant dynamin-related protein families DRP1 and DRP2 in plant development. Biochem. Soc. Trans. 38, 797-806 (2010).

43. Fujimoto, M. et al. Arabidopsis dynamin-related proteins DRP2B and DRP1A participate together in clathrin-coated vesicle formation during endocytosis. Proc. Natl Acad. Sci. USA 107, 6094-6099 (2010).

44. Konopka, C. A. \& Bednarek, S. Y. Comparison of the dynamics and functional redundancy of the arabidopsis dynamin-related isoforms DRP1A and DRP1C during plant development. Plant Physiol. 147, 1590-1602 (2008).

45. Kang, B. H., Busse, J. S. \& Bednarek, S. Y. Members of the Arabidopsis dynamin-like gene family, ADL1, are essential for plant cytokinesis and polarized cell growth. Plant Cell 15, 899-913 (2003).

46. Denk, W. \& Horstmann, H. Serial block-face scanning electron microscopy to reconstruct three-dimensional tissue nanostructure. PLoS Biol. 2, 1900-1909 (2004).

47. Behnke, H. D. Sieve elements - anucleate cells specialized for substancetransport in plants. Naturwissenschaften 77, 1-11 (1990)

48. van Bel, A. J. E. \& Knoblauch, M. Sieve element and companion cell: the story of the comatose patient and the hyperactive nurse. Aust. J. Plant Physiol. 27, 477-487 (2000)

49. Lee, J. Y. \& Lu, H. Plasmodesmata: the battleground against intruders. Trends Plant Sci. 16, 201-210 (2011).

50. Lucas, W. J., Yoo, B. C. \& Kragler, F. RNA as a long-distance information macromolecule in plants. Nat. Rev. Mol. Cell Biol. 2, 849-857 (2001).

51. Mullen, G. P. et al. Choline transport and de novo choline synthesis support acetylcholine biosynthesis in Caenorhabditis elegans cholinergic neurons. Genetics 177, 195-204 (2007)

52. Zufferey, R., Santiago, T. C., Brachet, V. \& Ben Mamoun, C. Reexamining the role of choline transporter-like (Ctlp) proteins in choline transport. Neurochem. Res. 29, 461-467 (2004).

53. Thompson, M. V. Phloem: the long and the short of it. Trends Plant Sci. 11, 26-32 (2006)

54. Thompson, M. V. \& Holbrook, N. M. Application of a single-solute nonsteady-state phloem model to the study of long-distance assimilate transport. J. Theor. Biol. 220, 419-455 (2003).

55. Nakata, K., Okuda, T. \& Misawa, H. Ultrastructural localization of high-affinity choline transporter in the rat neuromuscular junction: enrichment on synaptic vesicles. Synapse 53, 53-56 (2004).

56. Ribeiro, F. M. et al. Constitutive high-affinity choline transporter endocytosis is determined by a carboxyl-terminal tail dileucine motif. J. Neurochem. 94, 86-96 (2005).

57. Ribeiro, F. M. et al. The 'ins' and 'outs' of the high-affinity choline transporter CHT1. J. Neurochem. 97, 1-12 (2006).

58. Ribeiro, F. M. et al. Regulated recycling and plasma membrane recruitment of the high-affinity choline transporter. Eur. J. Neurosci. 26, 3437-3448 (2007).

59. Luu, D. T. \& Maurel, C. Aquaporin trafficking in plant cells: an emerging membrane-protein model. Traffic 14, 629-635 (2013).

60. Dhonukshe, P. et al. Clathrin-mediated constitutive endocytosis of PIN auxin efflux carriers in Arabidopsis. Curr. Biol. 17, 520-527 (2007) 
61. Dhonukshe, P. et al. Generation of cell polarity in plants links endocytosis, auxin distribution and cell fate decisions. Nature 456, 962-966 (2008).

62. Kleine-Vehn, J. et al. Recycling, clustering, and endocytosis jointly maintain PIN auxin carrier polarity at the plasma membrane. Mol. Syst. Biol. 7, 540 (2011).

63. Dettmer, J., Hong-Hermesdorf, A., Stierhof, Y. D. \& Schumacher, K. Vacuolar $\mathrm{H}+-$ ATPase activity is required for endocytic and secretory trafficking in Arabidopsis. Plant Cell 18, 715-730 (2006).

64. Arabidopsis Genome Initiative. Analysis of the genome sequence of the flowering plant Arabidopsis thaliana. Nature 408, 796-815 (2000).

65. Karimi, M., Inze, D. \& Depicker, A. GATEWAY vectors for Agrobacteriummediated plant transformation. Trends Plant Sci. 7, 193-195 (2002).

66. Clough, S. J. \& Bent, A. F. Floral dip: a simplified method for Agrobacteriummediated transformation of Arabidopsis thaliana. Plant J. 16, 735-743 (1998).

67. Lee, J. Y. et al. Transcriptional and posttranscriptional regulation of transcription factor expression in Arabidopsis roots. Proc. Natl Acad. Sci. USA 103, 6055-6060 (2006).

68. Mullendore, D. L., Windt, C. W., Van As, H. \& Knoblauch, M. Sieve tube geometry in relation to phloem flow. Plant Cell 22, 579-593 (2010).

69. Yang, Y., Hammes, U. Z., Taylor, C. G., Schachtman, D. P. \& Nielsen, E. Highaffinity auxin transport by the AUX1 influx carrier protein. Curr. Biol. 16, 1123-1127 (2006).

70. Deerinck, T. J., Bushong, E. A., Thor, A. \& Ellisman, M. H. A new protocol for preparation of biological specimens for serial block face scanning electron microscopy. Microscop. Microanal. 16, 1138-1139 (2010).

\section{Acknowledgements}

This work was supported by the grants by the Academy of Finland, Tekes and European Research Council (No.323052) (to Y.H.), Helsinki Graduate Program in Biotechnology and Molecular Biology (GPBM; to R.U.), the Japan Society for the Promotion of Science
(JSPS) Research Fellowships for Young Scientists (to S.M.), European Molecular Biology Organisation (EMBO, ALTF 450-2007; to J.D). IB and SBF-SEM are supported by Biocenter Finland. We thank Mervi Lindman and Antti Salminen for assistance with EM specimen preparation, François-Marie Meunier for the help with CTL phylogenetic tree Markus Grebe for sending the DRP1A-GFP and DRP1C-GFP lines and Jung-ok Heo for the help with graphical images. We thank Luis Herrera Estrella for sending the xipotl mutant seeds and Kerttuli Helariutta for the help with $\mathrm{C}^{14}$-labelled auxin transport experiments.

\section{Author contributions}

J.D., R.U., A.C. and S.M. carried out experimental work and wrote the paper. J.D., R.U. and A.C. contributed equally. I.B., S.R.Y. and E.J. performed the SBEM work, S.O. carried out the choline uptake experiments in Xenopus oocytes. D.L.M. and M.K. provided the Scanning Electron Microscopy data. C.L., K. S. and D.W. performed the whole-genome sequencing; A.P. provided the fluorescent choline derivative. Y.-D.S. analysed the CHER localization by using the immunogold labelling technique. T.M. carried out the metabolite profiling work.

\section{Additional information}

Supplementary Information accompanies this paper at http://www.nature.com/ naturecommunications

Competing financial interests: The authors declare no competing financial interests.

Reprints and permission information is available online at http://npg.nature.com/ reprintsandpermissions/

How to cite this article: Dettmer, J. et al. CHOLINE TRANSPORTER-LIKE1 is required for sieve plate development to mediate long distance cell-to-cell communication. Nat. Commun. 5:4276 doi: 10.1038/ncomms5276 (2014). 